\title{
Ultrastructural Evidence of Exosome Secretion by Progenitor Cells in Adult Mouse Myocardium and Adult Human Cardiospheres
}

\author{
Lucio Barile, ${ }^{1}$ Mihaela Gherghiceanu, ${ }^{2}$ Laurentiu M. Popescu, ${ }^{2}$ \\ Tiziano Moccetti, ${ }^{1}$ and Giuseppe Vassalli ${ }^{1,3}$ \\ ${ }^{1}$ Molecular Cardiology Laboratory, Fondazione Cardiocentro Ticino, Via Tesserete, 6900 Lugano, Switzerland \\ ${ }^{2}$ Ultrastructural Pathology, "Victor Babeş" National Institute of Pathology, 99-101 Spl. Independentei, 050096 Bucharest 5, Romania \\ ${ }^{3}$ Department of Cardiology, Centre Hospitalier Universitaire Vaudois (CHUV), Avenue du Bugnon, 1011 Lausanne, Switzerland
}

Correspondence should be addressed to Giuseppe Vassalli, giuseppe.vassalli@cardiocentro.org

Received 18 June 2012; Accepted 16 July 2012

Academic Editor: Ken-ichi Isobe

Copyright (C) 2012 Lucio Barile et al. This is an open access article distributed under the Creative Commons Attribution License, which permits unrestricted use, distribution, and reproduction in any medium, provided the original work is properly cited.

\begin{abstract}
The demonstration of beneficial effects of cell therapy despite the persistence of only few transplanted cells in vivo suggests secreted factors may be the active component of this treatment. This so-called paracrine hypothesis is supported by observations that culture media conditioned by progenitor cells contain growth factors that mediate proangiogenic and cytoprotective effects. Cardiac progenitor cells in semi-suspension culture form spherical clusters (cardiospheres) that deliver paracrine signals to neighboring cells. A key component of paracrine secretion is exosomes, membrane vesicles that are stored intracellularly in endosomal compartments and are secreted when these structures fuse with the cell plasma membrane. Exosomes have been identified as the active component of proangiogenic effects of bone marrow CD $34^{+}$stem cells in mice and the regenerative effects of embryonic mesenchymal stem cells in infarcted hearts in pigs and mice. Here, we provide electron microscopic evidence of exosome secretion by progenitor cells in mouse myocardium and human cardiospheres. Exosomes are emerging as an attractive vector of paracrine signals delivered by progenitor cells. They can be stored as an "off-the-shelf" product. As such, exosomes have the potential for circumventing many of the limitations of viable cells for therapeutic applications in regenerative medicine.
\end{abstract}

\section{Introduction}

Myocardial infarction and ensuing heart failure are the leading cause of mortality in Western countries. Infarction causes a massive loss of cardiomyocytes, which are replaced by scar tissue. To compensate for lost contractile cells, the remaining cardiomyocytes undergo hypertrophy and the heart remodels. These adaptive mechanisms are detrimental in the long run, eventually leading to congestive heart failure. Heart transplantation remains the ultimate treatment for chronic heart failure; however, this approach is limited by donor organ shortage, graft rejection, and the need for life-long immunosuppression.
Over the past decade, cell transplantation has been evaluated as a novel approach for heart failure. An early study reported that adult mouse bone marrow (BM) hematopoietic stem cells (HSCs) injected into infarcted mouse hearts differentiated into cardiomyocytes and improved cardiac function [1]. This report sparked widespread enthusiasm for BM cell transplantation as a potential approach for repairing broken hearts. Several subsequent studies failed to confirm the ability of HSCs to transdifferentiate into cardiomyocytes $[2,3]$. However, these negative results did not prevent clinical studies of autologous BM stem cell transplantation for cardiac regeneration from being initiated [4-9]. Multiple types of stem and progenitor cells, including embryonic 
stem cells (ESCs), adult skeletal myoblasts, adult BM-derived mononuclear cells, purified BM-derived subpopulations (e.g., c-kit ${ }^{+}$and $\mathrm{CD}_{133^{+}}$), and BM or adipose-derived mesenchymal stem cells (MSCs) have been evaluated as cell sources for heart cell therapy $[10,11]$.

Almost ten years after the initiation of randomized, controlled clinical trials of cell therapy for cardiac regeneration, it must be recognized that results have been inconsistent, and the overall improvement of cardiac function in patients after myocardial infarction (MI) has been modest [12-14]. The optimal timing of cell transfer, dose, delivery technique, the mechanism of action, patient selection, cell retention, and cell survival after transplantation are poorly understood. In addition, impaired cell functionality in old patients and in those with advanced cardiovascular disease or comorbidities limits autologous cell transplantation [15]. Hence, an unresolved paradox persists between robust benefits of cell therapy in animal models and modest effects in patients.

Transdifferentiation of adult BM cells into cardiomyocytes has not been demonstrated in humans. Originally proposed as an approach for providing injured hearts with new cells capable of restoring contractile function in scar areas, the biological rationale of cell therapy has progressively shifted toward beneficial effects mediated by transplanted cells on neighboring cells, possibly including the stimulation of endogenous regenerative mechanisms. Increasing evidence suggests cell therapy, whether myoblast or BM cellbased, may act by increasing the cellular mass in the infarcted area, thereby reducing ventricular remodeling, and by preventing cardiomyocytes in the ischemic peri-infarct zone from dying. The second mechanism, which involves secreted factors, has been referred to as the "paracrine hypothesis." Growth factors and cytokines secreted by transplanted cells activate endogenous intracellular signaling pathways potentially resulting in improved survival of endogenous cells and formation of new blood vessels [16-18].

\section{Adult Cardiac Stem Cells (CSCs) Expressing Stem Cell Surface Markers}

The adult myocardium is a highly organized tissue comprised of multiple cell types, including cardiomyocytes, endothelial cells, vascular smooth muscle cells, multiple types of interstitial cells, and extracellular matrix (ECM), which form a cardiovascular unit [19]. Interstitial cells include cardiacresident stem and progenitor cells (CSCs) located within stem cell niches [20]. These niches contain particular ECM components, supporting cells, nerves, and blood vessels. Several groups have isolated and characterized bona fide adult CSCs from rodents and humans [21-29]. Molecular markers that have been used to identify CSC populations include cell-surface epitopes expressed on stem cells in other tissues, particularly on HSCs, such as c-kit (CD117; the receptor for stem cell factor) and stem cell antigen-1 (Sca-1). At minimum, five seemingly different populations of CSCs have been described [30]. Whether these populations represent distinct entities of CSCs or different developmental stages or activation states of a single entity of CSC remains unclear. Likewise, the origin of CSCs has not been definitively established. In this regard, we and others have shown that BM-derived cells can acquire stem cell properties in the damaged heart $[31,32]$.

It has been speculated that cells derived from the heart itself may be a logical candidate cell source for repairing this organ, as these cells might be intrinsically programmed to support cardiac cell survival and function. In animal models of MI, injection of in vitro expanded CSCs has been associated with functional improvement [21-27]. However, direct comparisons of CSCs with other cell sources are needed in order to identify the most effective cell type. Recently, novel approaches based on adult cell reprogramming (induced pluripotent stem cells; iPS) have been developed [33]. Although extremely promising, these strategies are still associated with potential safety concerns. The discussion of these modalities is beyond the scope of the present work (the interested reader is referred to an excellent review published elsewhere [34]).

\section{Cardiospheres (CSs) and CS-Derived Cells (CDCs)}

First described in neural stem cells (neurospheres) [35], "spheres" have been considered-or named, at least-a feature of stemness. However, spheres can result not only from cell proliferation but also from cell aggregation, and therefore be clonal or nonclonal, respectively. The proportion of clonal spheres increases with decreasing cell density in a culture dish [36].

First described by Messina et al. [37], "cardiospheres" (CSs) are self-assembling spherical clusters of cells obtained by outgrowth from cardiac explants in the primary ex vivo tissue culture. CSs grow in semisuspension culture on polyD-lysine. They represent the best in vitro model of CSC niche-like environment [38]. While undifferentiated cells proliferate in the core of the CS, cardiac-committed cells grow on the periphery. We have generated CSs from the cellular outgrowth from adult human atrial appendage explants (Figure 1). The cellular outgrowth expressed MSC surface markers $\left(\mathrm{CD}_{13}{ }^{+}, \mathrm{CD}^{+} 3^{+}\right.$, and $\left.\mathrm{CD}_{105^{+}}\right)$but not hematopoietic markers $\left(\mathrm{CD}_{4} 5^{-}\right)$. In line with previous studies $[25,37]$, we have demonstrated the expression of cardiac-specific genes, such as troponin I, in human CSs (Figure 2). CSs placed in a new culture dish disassembled and gave rise to a monolayer of CS-derived cells (CDCs) that formed secondgeneration CSs. CDCs could be expanded as monolayers on fibronectin (Figure 1). Previous studies [25, 39] reported that CDCs are clonogenic and have multilineage differentiation potential. By contrast, a recent study in rodents questioned the notion of CSs as a source of stem cells with cardiomyogenic potential [40]. This study suggested spontaneously beating CSs may result from remnants of myocardial tissue in the cellular outgrowth from cardiac explants. Regardless of this issue, CDCs have been shown to improve left ventricular ejection fraction (LVEF) in SCID beige mice 3 weeks after MI when compared to mice injected with vehicle or with adult normal human dermal fibroblasts [25]. These beneficial effects were associated with increased blood vessel formation and decreased apoptosis [41]. 

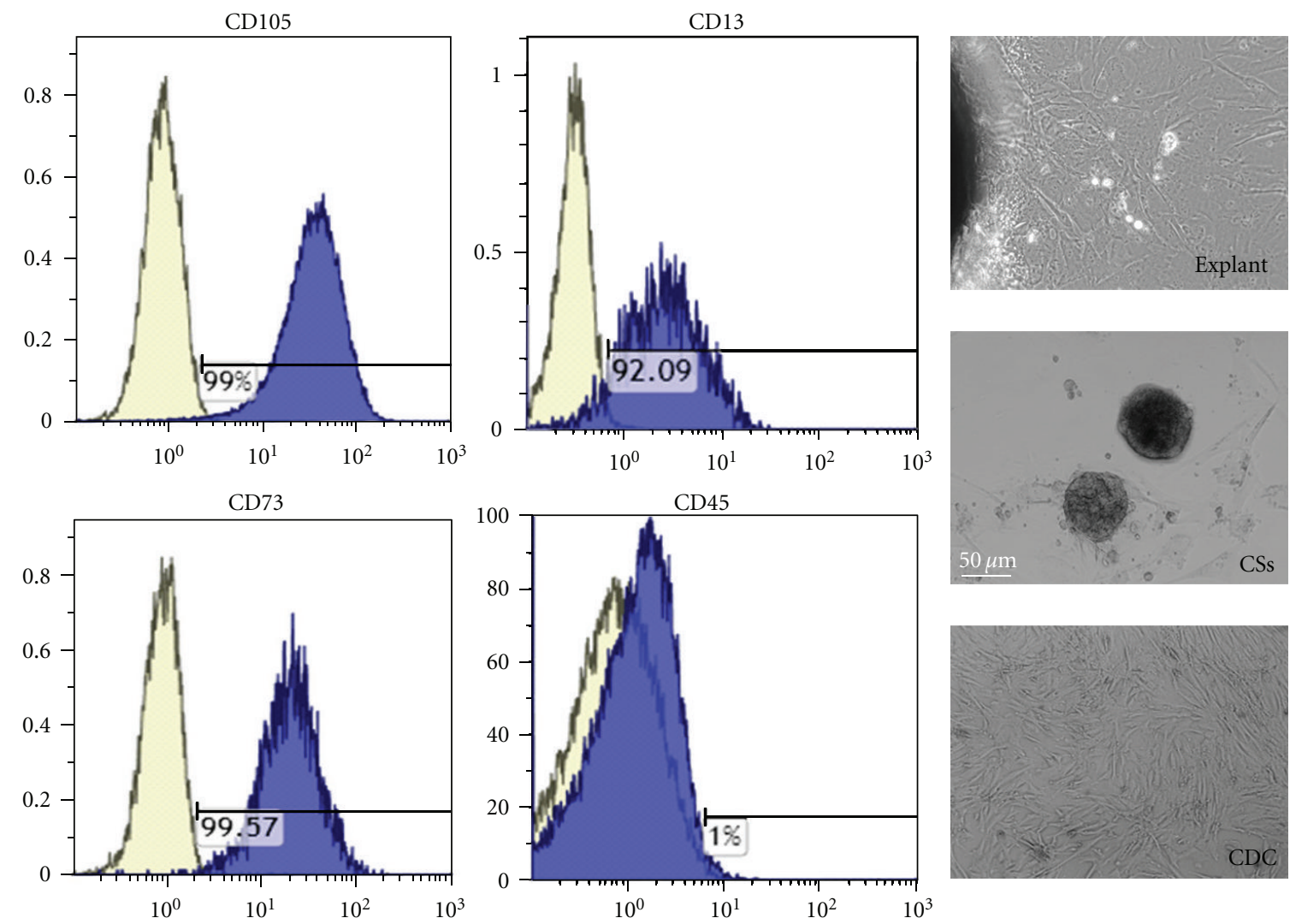

FiguRE 1: Left and middle panels: Flow cytometric analysis of the cellular outgrowth from a human atrial appendage explant in the primary tissue culture, showing $\mathrm{MSC}\left(\mathrm{CD} 13^{+}, \mathrm{CD} 3^{+}\right.$, and $\left.\mathrm{CD} 105^{+}\right)$but not hematopoietic $\left(\mathrm{CD} 45^{-}\right)$marker expression. Upper right panel: Photomicrograph of a human atrial explant with cellular outgrowth; middle right panel: human CSs; lower right panel: human CDCs.

Autologous c-kit ${ }^{+}$, differentiation lineage-negative $\left(\mathrm{Lin}^{-}\right)$CSCs, as well as CDCs, have recently been tested in initial clinical studies. These two clinical trials of CSC therapy for cardiac repair are briefly discussed in the next section.

\section{Clinical Studies of Autologous CSCs for Ischemic Heart Disease}

The first clinical trial of autologous CSCs for ischemic heart disease was the stem cell infusion in patients with ischemic cardiomyopathy (SCIPIO) trial (registered with ClinicalTrials.gov, number NCT00474461) [42]. This phase-1 clinical trial tested autologous $\mathrm{c}-\mathrm{kit}^{+} / \mathrm{Lin}^{-}$CSCs for treatment of heart failure resulting from ischemic heart disease. In stage A of this trial, patients with reduced LVEF $(\leq 40 \%)$ after MI before coronary artery bypass grafting (CABG) were consecutively enrolled in the treatment and control groups. In stage $\mathrm{B}$, patients were randomly assigned to the treatment or control group. Autologous CSCs were administered by intracoronary infusion at a mean of 113 days (SE 4) after surgery. Sixteen patients were assigned to the treatment group and seven to the control group. No cell therapy-related adverse effects were reported. In 14 CSC-treated patients who were analyzed, LVEF increased from 30.3\% (SE 1.9) before CSC infusion to $38.5 \%$ (SE 2.8) at 4 months after infusion
$(P=0.001)$. Conversely, in seven control patients LVEF did not change during the corresponding time interval $(30.1 \%$ [SE 2.4] at 4 months after CABG versus 30.2\% [SE 2.5] at 8 months after $\mathrm{CABG}$ ). In eight treated patients studied at 1 year, LVEF increased by 12.3 LVEF units [SE 2.1] versus baseline $(P=0.0007)$. These results suggest intracoronary infusion of autologous c-kit ${ }^{+} / \mathrm{Lin}^{-}$CSCs may enhance LV systolic function in patients with heart failure after MI. In the seven treated patients in whom cardiac magnetic resonance imaging (MRI) was done, infarct size decreased from $32.6 \mathrm{~g}$ (SE 6.3 ) by $7.8 \mathrm{~g}(\mathrm{SE} 1.7 ; 24 \%)$ at 4 months $(P=0.004)$ and $9.8 \mathrm{~g}(\mathrm{SE} 3.5 ; 30 \%)$ at 1 year $(P=0.04)$. This reduction in scar is difficult to interpret due to the lack of MRI data in the control group.

The prospective, randomized CArdiosphere-Derived aUtologous stem CElls to reverse ventricUlar dySfunction (CADUCEUS) trial (registered with ClinicalTrials.gov, NCT00893360) evaluated autologous CDCs in patients with reduced LVEF $(=25-45 \%$; mean baseline value $=39 \%$; $[\mathrm{SD}$ 12]) 2-4 weeks after AMI [43]. Seventeen patients received CDCs and eight patients were randomized to the control group. Autologous CDCs grown from endomyocardial biopsy specimens were infused into the infarct-related artery 1.5-3 months after MI. Biopsy samples yielded the prescribed cell doses within 36 days (SD 6). By 6 months, no patients had died, developed cardiac tumors, or major 


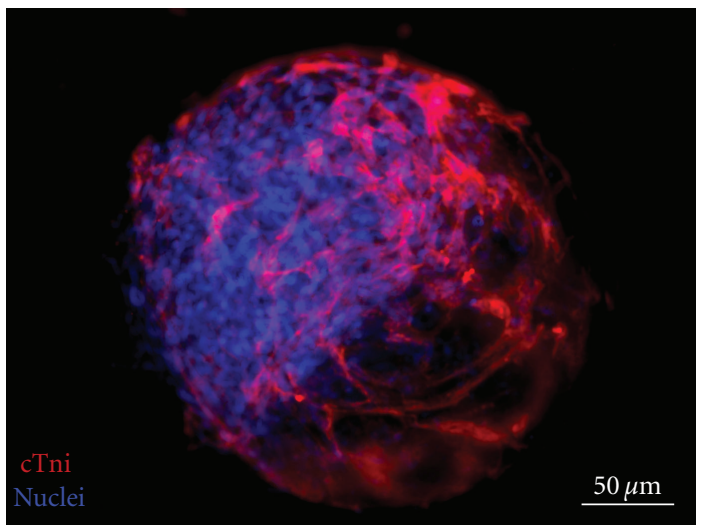

FIGURE 2: Immunostaining of a human CS showing expression of cardiac troponin I (red); nuclei stain blue.

adverse cardiac events in either group. Four patients (24\%) in the CDC group had serious adverse events compared with one control (13\%; $P=1.00)$. MRI analysis showed reductions in scar mass by $28 \%$ by 6 months and $42 \%$ by 12 months $(P=0.001)$, increases in viable heart mass by $13.0 \mathrm{~g}$ at 6 months $(P=0.01)$, greater regional contractility $(-11.8 \%$ versus $-8.5 \% ; P=0.02)$, and regional systolic wall thickening $(P=0.015)$ in the CDC group compared with controls. Changes in end-diastolic volume, end-systolic volume, and LVEF did not differ between groups by 6 months. These results suggest that intracoronary infusion of autologous $\mathrm{c}-\mathrm{kit}^{+} / \mathrm{Lin}^{-}$CSCs or CDCs after myocardial infarction is safe and might be beneficial. Larger clinical trials to test the efficacy of these approaches are being planned.

\section{Paracrine Effects of Transplanted Cells}

Based on the number of human-specific cells relative to overall increases in capillary density and myocardial viability, Chimenti et al. [41] estimated that direct progenitor cell differentiation quantitatively accounted for $20 \%$ to $50 \%$ of the observed effects of human CDCs transplanted into infarcted SCID mouse hearts. Conversely, a large part of these effects seemed attributable to endogenous cells. In vitro, culture media conditioned by human adult CDCs prevented apoptosis in neonatal rat ventricular myocytes under hypoxic conditions, while promoting angiogenesis from human umbilical vein endothelial cells (HUVECs). In vivo, human CDCs secreted hepatocyte growth factor-1 (HGF-1), insulin-like growth factor-1 (IGF-1), and vascular endothelial growth factor (VEGF) when transplanted into the same SCID mouse model of MI, where they were shown to induce tissue regeneration and improve function. Injection of CDCs into the peri-infarct zone increased the expression of the prosurvival factor Akt, reduced apoptosis, and increased capillary density. Although myocardial perfusion was not directly measured in this study, increased capillary density was consistent with a role for angiogenesis in functional improvement. These findings were interpreted as evidence for paracrine effects of CDCs exceeding those of direct regeneration. In another study, Tang et al. [44] reported beneficial effects of paracrine factors secreted by rat c-kit ${ }^{+}$
CSCs delivered by intracoronary infusion 4 weeks after MI. Although no engrafted donor cells were found in some hearts in the cell therapy group, scar area was reduced and cardiac output increased compared with the control group. The number of cardiomyocytes, blood vessels, and endogenous cardiac progenitor cells was increased in hearts injected with c-kit ${ }^{+}$CSCs, even in the case when no engrafted cells were observed. Smits et al. [45] showed that injection of human cardiac progenitor cells into infarcted mouse hearts reduced cardiac remodeling 3 months after MI, despite the fact that only $3-4 \%$ of the injected cells could be found in the hearts at this time point. Together, these findings suggest factors released by CSCs may mediate sustained beneficial effects, including angiogenesis and improved cardiomyocyte survival. In this regard, CDCs have been shown to secrete a number of growth factors [41] and microRNAs (miRNAs) [46] that regulate intracellular signaling pathways in neighboring cells. Culture media conditioned by ESCs or MSCs were shown to improve myocardial function after ischemia by reducing apoptosis and infarct size both in vitro and in vivo [47-49]. Additional types of interstitial cells including resident cardiac immune cells [50] may also contribute to the secretion of cytokines and growth factors into their microenvironment. Collectively, these studies indicate that paracrine effects of progenitor cells are a central mechanism of cell therapy. This notion implies a dispensable role for cell transplantation in therapeutic approaches for cardiac regeneration [17].

\section{Microparticles and Exosomes}

The demonstration of beneficial effects of cell therapy despite short-lived survival of the delivered cells, along with the observed trophic effects of culture media conditioned by progenitor cells, suggests that secreted factors may be the active component of cell therapy for cardiac regeneration, as mentioned. Cells communicate with each other via released molecules such as short peptides, proteins, nucleotides, and lipids that bind to surface receptors on neighboring cells. In addition, eukaryotic cells communicate with each other through the release of microparticles and exosomes in their extracellular environment. Microparticles are 
a heterogeneous population of spherical structures with a diameter of 100-1000 nm, which are released by budding of the plasma membrane (ectocytosis) as phospholipid vesicles that express antigens specific of their parental cells [51]. Circulating microparticles are increased in a number of disease conditions, such as inflammatory and autoimmune diseases, atherosclerosis, and cancer. Distinct from microparticles, exosomes are membrane vesicles with a diameter of 40 $100 \mathrm{~nm}$, formed by endocytosis, a process that involves the sequestration of plasma membrane proteins within the exosomes. Exosomes are stored intracellularly in endosomal compartments and are secreted when these multivesicular structures fuse with the cell plasma membrane [52-55].

Exosomes display a broad spectrum of bioactive substances on their surface and carry a concentrated set of proteins, lipids, and even nucleic acids that are taken up by other cells and regulate their function [54-56]. Some exosomal membrane proteins are cleaved by proteases, and the resulting fragments may act as ligands for cell surface receptors in the target cell. Exosomes can also transfer exosomal proteins and RNA nonselectively by fusing with target cells. They are released by many cell types, including dendritic cells, mast cells, B and T cells, platelets, neurons, tumor cells, and MSCs.

Exosomes do not carry a random array of the intracellular proteins but a specific set of proteins derived from the plasma membrane, endocytic pathway, and the cytosol, with only low amounts of proteins from other intracellular compartments $[53,56]$. Most exosomes contain tetraspanins (CD81, CD63, and CD9) which play important roles in cell penetration, invasion and fusion events, as well as multivesicular body molecules (Alix, Tsg101, and clathrin), heat shock protein 70 (HSP70), and Rabs that regulate exosome docking and membrane fusion [57]. Exosomes also contain annexins, metabolic enzymes, ribosomal proteins, signal transduction molecules, adhesion molecules, ATPases, cytoskeletal and ubiquitin molecules, growth factors, cytokines, mRNA, and microRNA (miRNA) molecules $[53,56,58]$. An exosome protein and RNA database (ExoCarta) is available at http://exocarta.ludwig.edu.au/. In addition to molecules shared by exosomes from multiple cell types, exosomes carry specific proteins from their parental cell type. It has been shown that mRNAs carried by exosomes can be translated into proteins in the target cell, indicating that exosomes can act as a vector of genetic information. ESC-derived microvesicles have been shown to reprogram hematopoietic progenitors by mRNA transfer and protein delivery [59]. miRNA families can be selectively secreted into the extracellular environment via exosomes [60]. Dendritic cells release exosomes that are loaded with distinct sets of miRNA dependent on the status of dendritic cell activation [61]. These exosomes fuse with target cells, thereby transferring miRNAs that can repress mRNAs in these cells. Thus, exosomes can mediate epigenetic effects by transferring specific miRNA molecules between cells.

Biological effects of exosomes are cell type-specific, reflecting their molecular composition. Recently, Sahoo et al. [62] reported angiogenic effects of exosomes derived from human $\mathrm{CD}_{3} 4^{+} \mathrm{BM}$ stem cells in isolated endothelial cells and in murine models of vessel growth. These exosomes were enriched with proangiogenic miRNAs. By contrast, exosomes derived from CD34-depleted BM cells lacked angiogenic activities. In some of the in vitro and in vivo assays, the exosomes from $\mathrm{CD} 34^{+}$cells appeared more potent than the cells themselves, possibly as a result of the durability of the exosome in culture. Vrijsen et al. [63] reported that exosomes mediated the angiogenic activity of media conditioned by human fetal cardiac progenitor cells in vitro. Timmers et al. [49] showed that injection of conditioned medium from ESC-derived MSCs reduced infarct size and improved cardiac function in a pig model of ischemia/reperfusion, as mentioned above. Exosomes within the conditioned medium were shown to contain the active component [64]. Lai et al. [65] found that exosomes secreted by MSCs similarly reduced myocardial ischemia/reperfusion injury in mice.

In extracardiac biological systems, exosomes were shown to secrete synuclein that impacted neuronal survival [66], or $\alpha \mathrm{B}$ crystallin from polarized human retinal pigment epithelium, which provided neuroprotection to adjacent cells [67], just to mention a few examples. Altogether, exosomes act as vectors for the intercellular exchange of biological signals and information, which mediate cell activation, phenotypic changes, and reprogramming of cell function. Exosomes and microparticles represent a transcellular delivery system that expands the limited transcriptome and proteome of recipient cells and establishes a communication network among cells [51].

\section{Ultrastructural Evidence of Exosome Secretion by Cardiac Progenitor Cells}

Since CDCs deliver beneficial paracrine signals to injured myocardium [41], and since exosomes have been identified as the active component of the paracrine effects of $\mathrm{CD} 34^{+}$ HSCs [62] or MSCs $[64,65]$ in infarcted hearts, we investigated whether CSCs and human CSs secrete exosomes. Figure 3 shows transmission electron micrographs of progenitor cells within a stem cell niche in a mouse adult heart, as well as in an adult human CS in vitro. Progenitor cells exhibit a similar ultrastructural appearance in the two contexts. Here, we also provide, for the first time, ultrastructural evidence of exosome and microvesicle secretion by progenitor cells in mouse adult heart and in human CSs (Figure 4$)$. Microvesicles ( $\approx 200 \mathrm{~nm}$ diameter) have an electron-dense content. Exosomes can be distinguished from microvesicles, apoptotic bodies, and other types of membrane vesicles by size and ultrastructural appearance. Exosomes and microvesicles seems to be an important mechanism involved in the heterocellular communication in the adult heart [68], especially between telocytes [69] and resident progenitor cells $[68,70]$. Some of us recently showed that exosomes emerge from telocytes in the border zone of myocardial infarction [71], consistent with a potential role for this secreted component in neoangiogenesis; however, a quantitative analysis was not performed.

Transmission electron micrographs suggesting exosome uptake by cardiomyocytes in the adult mouse heart are 


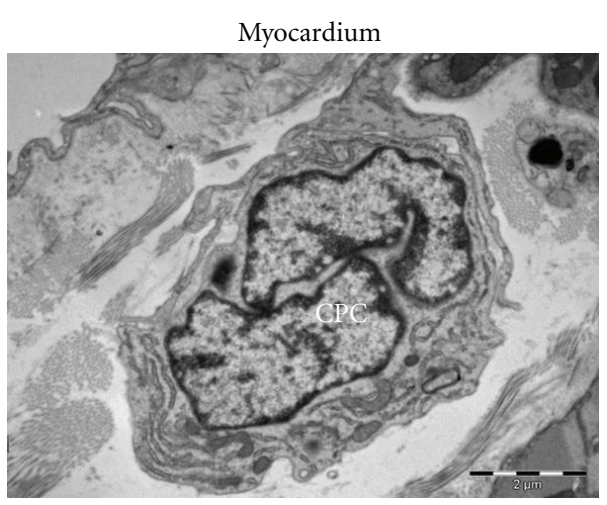

(a)

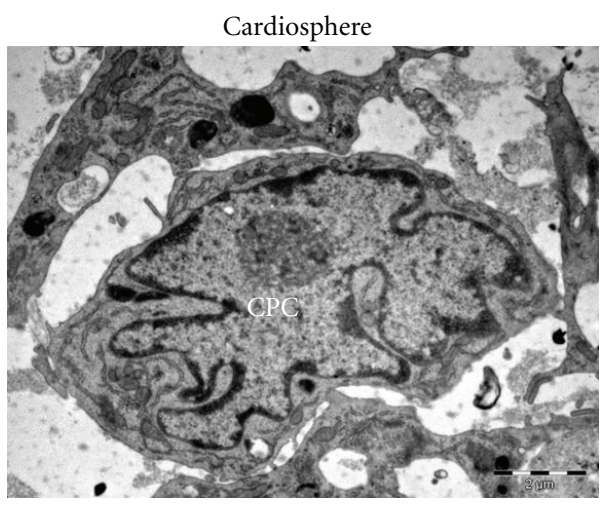

(b)

FIGURE 3: Transmission electron micrographs showing a cardiac progenitor cell (CPC), characterized by a large nucleus and a thin cytoplasmic rim, in adult mouse myocardium (a) and in an adult human CS in vitro (b).

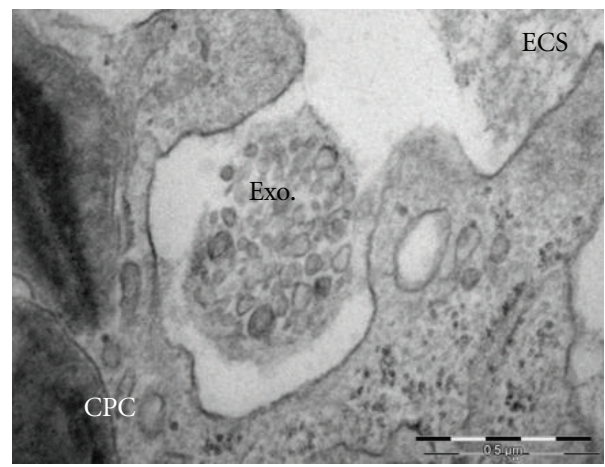

(a)

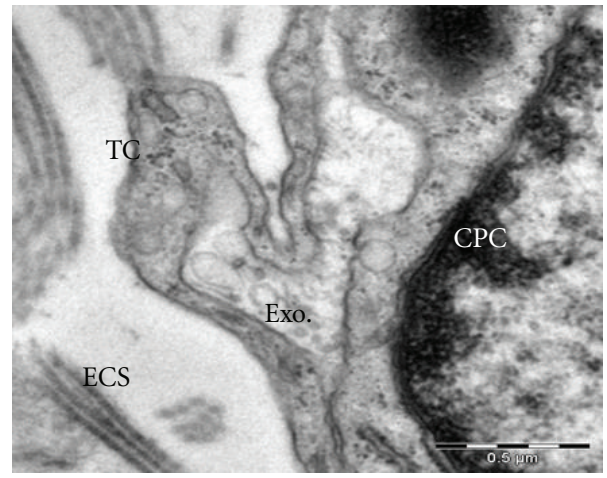

(c)

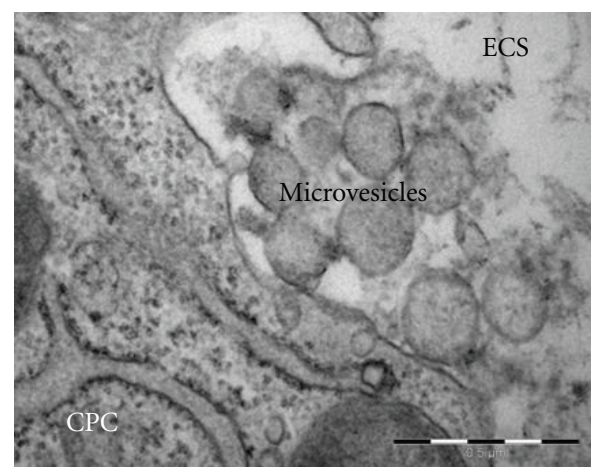

(b)

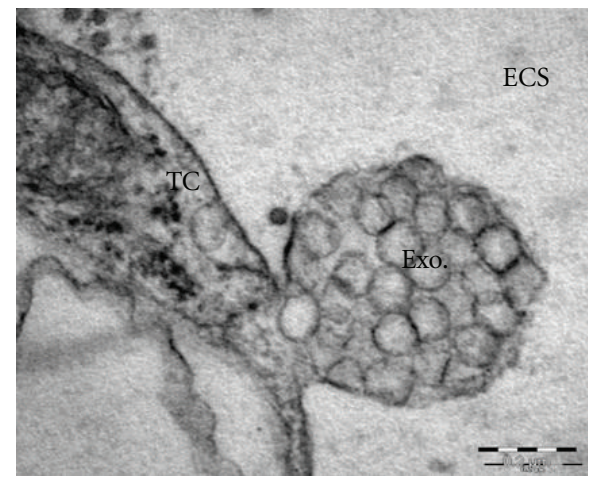

(d)

FIgURE 4: Transmission electron micrographs showing exosomes $(\approx 75 \mathrm{~nm}$ diameter; Exo. (a) $)$ and microvesicles $(\approx 200 \mathrm{~nm}$ diameter; $(\mathrm{b}))$ secreted by cardiac progenitor cells (CPC) in a human CS in vitro. Exosomes in adult mouse myocardium ((c)-(d); TC: telocyte; ESC: extracellular space).

shown in Figure 5. Cardiomyocytes seem to encircle exosomes on their cell surfaces with thin cytoplasmic processes, and exosomes are then incorporated into cardiomyocytes. However, this preliminary observation needs to be analysed in a larger number of samples. The molecular content and functional activities of exosomes secreted by human CSCs and CSs also remain to be characterized. An additional, unanswered question relates to changes in exosome secretion under normal and ischemic conditions.

\section{Therapeutic Potential of Exosomes}

Exosomes may circumvent many of the hurdles associated with the use of replicating cells as a therapeutic agent, such as the risk that replicating cells may increase in an uncontrolled manner over time or exert persisting biological activities regardless of the clinical needs of the patient. Also, many autologous stem cell therapies require ex vivo cell expansion, and therefore the preparation of the therapeutic 


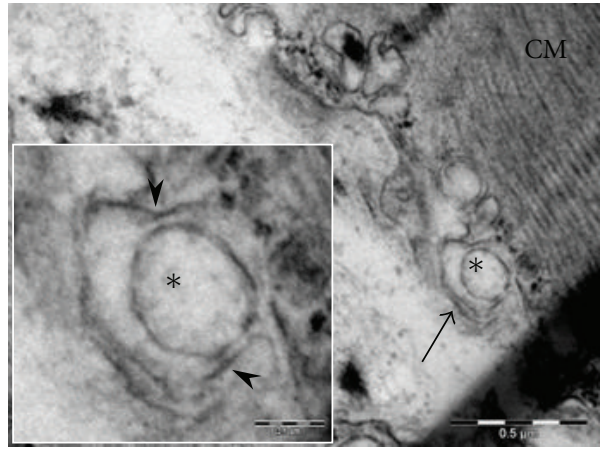

(a)

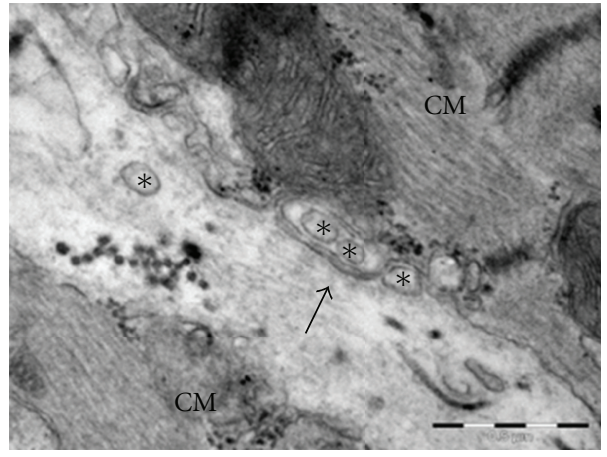

(b)

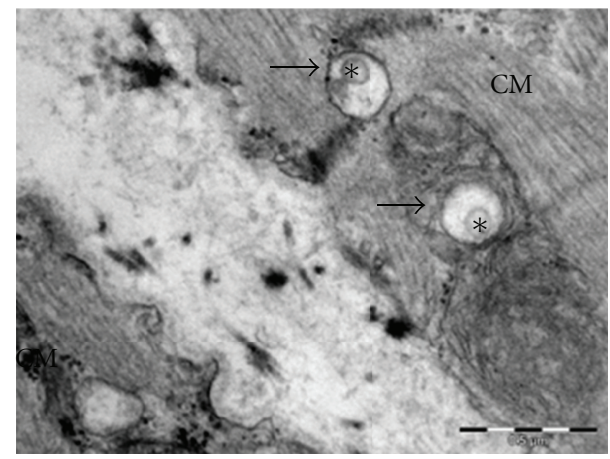

(c)

FIGURE 5: Transmission electron micrographs showing exosomes in an adult mouse heart. (a) Exosomes (asterisks) located at the cell surface of a cardiomyocyte (CM). The latter extends thin cytoplasmic processes (arrow) that encircle the exosome. Inset: higher magnification view showing dense nanostructures (arrowheads) at the interface of the CM membrane and the membrane of the exosome, suggesting involvement of molecular interactions in exosome uptake by CMs. (b) Exosomes (asterisks) encircled by cytoplasmic processes (arrow). (c) Exosomes (asterisks) taken up by the CM within small, round cytoplasmic structures (arrows).

product may take several weeks. Moreover, autologous cells from aged patients have limited regenerative potential [15], whereas allogeneic cells are generally rejected by the immune system (with the possible exception of MSCs, which mediate immunomodulatory effects [72]). A hypothetical advantage of exosomes is that it might be possible to use allogeneic cells from young, healthy individuals for their production, although this remains to be demonstrated. Exosomes are supposedly less immunogenic than their parental cells owing to a lower content of membrane-derived proteins including major histocompatibility complex (MHC) molecules. However, this may depend on the parental cell type. Since exosomes secreted by dendritic cells can transfer MHC class II molecules and stimulate immune cells [73], they have been tested in clinical trials in cancer patients [74-76]. CDCs have been shown to express MHC class I but not class II molecules [77], and therefore exosomes secreted by CDCs can be assumed to lack MHC class II. If alloimmunogenicity of exosomes secreted by CSs or CDCs turns out to be negligible, it will be possible to develop "off-the-shelf" therapies based on exosomes from young, healthy donors. Obviously, cell-free products offer a number of advantages compared with cell transplantation. Cell-free products can be standardized and tested in terms of dose and biological activity. Exosomes could be stored without potentially toxic cryopreservatives at $-20^{\circ} \mathrm{C}$ for 6 months with no loss in their biochemical activities [64]. Importantly, exosomes have been shown to protect their contents from degradation in vivo $[58,78]$, thereby potentially preventing some of the problems associated with small soluble molecules such as cytokines, growth factors, transcription factors, and RNAs, which are rapidly degraded. The durability of the exosomes in culture permits to achieve high doses of exosomes through collection from culture medium in which exosomes are secreted over periods of time [62]. Scalable systems for exosome production to support large-scale, commercially viable manufacturing processes have been described [64]. Exosomes therefore exhibit several attractive features as a therapeutic agent. On the other hand, potential limitations should be considered as well. For example, exosomes contain a mixture of biologically active molecules, some of which seem to have beneficial effects, whereas others might have detrimental (e.g., proinflammatory) effects under certain conditions. Whether exosomes will be superior to angiogenic drugs or purified, recombinant growth factors and other peptides within the context of cell-free approaches for tissue regeneration remains to be seen.

\section{Conclusions}

CSs represent an in vitro model that recapitulates several aspects of cell-cell interactions between CSCs and other 
cells in the heart. Exosomes may be a key mechanism by which cardiac progenitors communicate with each other and deliver paracrine signals to neighboring cells. Here, we provide, for the first time, ultrastructural evidence of exosome secretion by progenitor cells in the adult mouse heart, as well as in human CSs in vitro. Earlier studies have demonstrated beneficial effects of exosomes secreted by HSCs and MSCs in animal models of MI. These studies suggest exosomes may offer major advantages as a cell-free therapeutic product for cardiac regeneration.

\section{Authors' Contribution}

L. Barile and M. Gherghiceanu contributed equally to this work.

\section{Acknowledgments}

Support by the Swiss National Science Foundation, the Cecilia Augusta Foundation, the METIS Foundation Sergio Mantegazza, and the "Fondazione per la ricerca sulla trasfusione e sui trapianti” (Lugano) is gratefully acknowledged.

\section{References}

[1] D. Orlic, J. Kajstura, S. Chimenti et al., "Bone marrow cells regenerate infarcted myocardium," Nature, vol. 410, no. 6829, pp. 701-705, 2001.

[2] L. B. Balsam, A. J. Wagers, J. L. Christensen, T. Kofidis, I. L. Weissmann, and R. C. Robbins, "Haematopoietic stem cells adopt mature haematopoietic fates in ischaemic myocardium," Nature, vol. 428, no. 6983, pp. 668-673, 2004.

[3] C. E. Murry, M. H. Soonpaa, H. Reinecke et al., "Haematopoietic stem cells do not transdifferentiate into cardiac myocytes in myocardial infarcts," Nature, vol. 428, no. 6983, pp. 664 668, 2004.

[4] C. Stamm, B. Westphal, H. D. Kleine et al., "Autologous bonemarrow stem-cell transplantation for myocardial regeneration," The Lancet, vol. 361, no. 9351, pp. 45-46, 2003.

[5] K. C. Wollert, G. P. Meyer, J. Lotz et al., "Intracoronary autologous bone-marrow cell transfer after myocardial infarction: the BOOST randomised controlled clinical trial," The Lancet, vol. 364, no. 9429, pp. 141-148, 2004.

[6] V. Schächinger, S. Erbs, A. Elsässer et al., "Intracoronary bone marrow-derived progenitor cells in acute myocardial infarction," The New England Journal of Medicine, vol. 355, no. 12, pp. 1210-1221, 2006.

[7] B. Assmus, J. Honold, V. Schächinger et al., "Transcoronary transplantation of progenitor cells after myocardial infarction," The New England Journal of Medicine, vol. 355, no. 12, pp. 1222-1232, 2006.

[8] S. Janssens, C. Dubois, J. Bogaert et al., "Autologous bone marrow-derived stem-cell transfer in patients with STsegment elevation myocardial infarction: double-blind, randomised controlled trial," The Lancet, vol. 367, no. 9505, pp. 113-121, 2006.

[9] K. Lunde, S. Solheim, S. Aakhus et al., "Intracoronary injection of mononuclear bone marrow cells in acute myocardial infarction," The New England Journal of Medicine, vol. 355, no. 12, pp. 1199-1209, 2006.

[10] E. Forte, I. Chimenti, L. Barile et al., "Cardiac cell therapy: the next (re)generation," Stem Cell Reviews and Reports, vol. 7, pp. 1018-1030, 2011.
[11] E. Chavakis, M. Koyanagi, and S. Dimmeler, "Enhancing the outcome of cell therapy for cardiac repair: progress from bench to bedside and back," Circulation, vol. 121, no. 2, pp. 325-335, 2010.

[12] A. Abdel-Latif, R. Bolli, I. M. Tleyjeh et al., "Adult bone marrow-derived cells for cardiac repair: a systematic review and meta-analysis," Archives of Internal Medicine, vol. 167, no. 10, pp. 989-997, 2007.

[13] K. C. Wollert and H. Drexler, "Cell therapy for the treatment of coronary heart disease: a critical appraisal," Nature Reviews Cardiology, vol. 7, no. 4, pp. 204-215, 2010.

[14] D. M. Leistner, U. Fischer-Rasokat, J. Honold et al., "Transplantation of progenitor cells and regeneration enhancement in acute myocardial infarction (TOPCARE-AMI): final 5-year results suggest long-term safety and efficacy," Clinical Research in Cardiology, vol. 100, no. 10, pp. 925-934, 2011.

[15] S. Dimmeler and A. Leri, "Aging and disease as modifiers of efficacy of cell therapy," Circulation Research, vol. 102, no. 11, pp. 1319-1330, 2008.

[16] H. Ebelt, M. Jungblut, Y. Zhang et al., "Cellular cardiomyoplasty: improvement of left ventricular function correlates with the release of cardioactive cytokines," Stem Cells, vol. 25, no. 1, pp. 236-244, 2007.

[17] K. R. Vrijsena, S. A. J. Chamuleaua, W. A. Noorta, P. A. Doevendansa, and J. P. G. Sluijtera, "Stem cell therapy for endstage heart failure: indispensable role for the cell?" Current Opinion in Organ Transplantation, vol. 14, no. 5, pp. 560-565, 2009.

[18] M. Gnecchi, Z. Zhang, A. Ni, and V. J. Dzau, "Paracrine mechanisms in adult stem cell signaling and therapy," Circulation Research, vol. 103, no. 11, pp. 1204-1219, 2008.

[19] S. Ausoni and S. Sartore, "The cardiovascular unit as a dynamic player in disease and regeneration," Trends in Molecular Medicine, vol. 15, no. 12, pp. 543-552, 2009.

[20] K. Urbanek, D. Cesselli, M. Rota et al., "Stem cell niches in the adult mouse heart," Proceedings of the National Academy of Sciences of the United States of America, vol. 103, no. 24, pp. 9226-9231, 2006.

[21] A. P. Beltrami, L. Barlucchi, D. Torella et al., "Adult cardiac stem cells are multipotent and support myocardial regeneration," Cell, vol. 114, no. 6, pp. 763-776, 2003.

[22] H. Oh, S. B. Bradfute, T. D. Gallardo et al., "Cardiac progenitor cells from adult myocardium: homing, differentiation, and fusion after infarction," Proceedings of the National Academy of Sciences of the United States of America, vol. 100, no. 21, pp. 12313-12318, 2003.

[23] H. C. Ott, T. S. Matthiesen, J. Brechtken et al., "The adult human heart as a source for stem cells: repair strategies with embryonic-like progenitor cells," Nature Clinical Practice Cardiovascular Medicine, vol. 4, supplement 1, pp. S27-S39, 2007.

[24] C. Bearzi, M. Rota, T. Hosoda et al., "Human cardiac stem cells," Proceedings of the National Academy of Sciences of the United States of America, vol. 104, no. 35, pp. 14068-14073, 2007.

[25] R. R. Smith, L. Barile, H. C. Cho et al., "Regenerative potential of cardiosphere-derived cells expanded from percutaneous endomyocardial biopsy specimens," Circulation, vol. 115, no. 7, pp. 896-908, 2007.

[26] A. Linke, P. Müller, D. Nurzynska et al., "Stem cells in the dog heart are self-renewing, clonogenic, and multipotent and regenerate infarcted myocardium, improving cardiac function," Proceedings of the National Academy of Sciences of the United States of America, vol. 102, no. 25, pp. 8966-8971, 2005. 
[27] A. M. Smits, P. van Vliet, C. H. Metz et al., "Human cardiomyocyte progenitor cells differentiate into functional mature cardiomyocytes: an in vitro model for studying human cardiac physiology and pathophysiology," Nature Protocols, vol. 4, no. 2, pp. 232-243, 2009.

[28] L. Barile, I. Chimenti, R. Gaetani et al., "Cardiac stem cells: isolation, expansion and experimental use for myocardial regeneration," Nature Clinical Practice Cardiovascular Medicine, vol. 4, supplement 1, pp. S9-S14, 2007.

[29] L. Barile, E. Messina, A. Giacomello, and E. Marbán, "Endogenous cardiac stem cells," Progress in Cardiovascular Diseases, vol. 50, no. 1, pp. 31-48, 2007.

[30] G. M. Ellison, V. Galuppo, C. Vicinanza et al., "Cardiac stem and progenitor cell identification: different markers for the same cell?" Frontiers in Bioscience, vol. 2, pp. 641-652, 2010.

[31] L. Barile, F. Cerisoli, G. Frati et al., "Bone marrow-derived cells can acquire cardiac stem cells properties in damaged heart," Journal of Cellular and Molecular Medicine, vol. 15, no. 1, pp. 63-71, 2011.

[32] S. S. Fazel, L. Chen, D. Angoulvant et al., "Activation of ckit is necessary for mobilization of reparative bone marrow progenitor cells in response to cardiac injury," The FASEB Journal, vol. 22, no. 3, pp. 930-940, 2008.

[33] L. W. van Laake, L. Qian, P. Cheng et al., "Reporter-based isolation of induced pluripotent stem cell-and embryonic stem cell-derived cardiac progenitors reveals limited gene expression variance," Circulation Research, vol. 107, no. 3, pp. 340347,2010 .

[34] Y. Yoshida and S. Yamanaka, "IPS cells: a source of cardiac regeneration," Journal of Molecular and Cellular Cardiology, vol. 50, no. 2, pp. 327-332, 2011.

[35] B. A. Reynolds and S. Weiss, "Generation of neurons and astrocytes from isolated cells of the adult mammalian central nervous system," Science, vol. 255, no. 5052, pp. 1707-1710, 1992.

[36] E. Pastrana, V. Silva-Vargas, and F. Doetsch, "Eyes wide open: a critical review of sphere-formation as an assay for stem cells," Cell Stem Cell, vol. 8, no. 5, pp. 486-498, 2011.

[37] E. Messina, L. De Angelis, G. Frati et al., "Isolation and expansion of adult cardiac stem cells from human and murine heart," Circulation Research, vol. 95, no. 9, pp. 911-921, 2004.

[38] T. S. Li, K. Cheng, S. T. Lee et al., "Cardiospheres recapitulate a niche-like microenvironment rich in stemness and cell-matrix interactions, rationalizing their enhanced functional potency for myocardial repair," Stem Cells, vol. 28, no. 11, pp. 20882098, 2010.

[39] D. R. Davis, Y. Zhang, R. R. Smith et al., "Validation of the cardiosphere method to culture cardiac progenitor cells from myocardial tissue," PLoS ONE, vol. 4, no. 9, Article ID e7195, 2009.

[40] D. C. Andersen, P. Andersen, M. Schneider, H. B. Jensen, and S. P. Sheikh, "Murine "cardiospheres" are not a source of stem cells with cardiomyogenic potential," Stem Cells, vol. 27, no. 7, pp. 1571-1581, 2009.

[41] I. Chimenti, R. R. Smith, T. S. Li et al., "Relative roles of direct regeneration versus paracrine effects of human cardiospherederived cells transplanted into infarcted mice," Circulation Research, vol. 106, no. 5, pp. 971-980, 2010.

[42] R. Bolli, A. R. Chugh, D. D’Amario et al., "Cardiac stem cells in patients with ischaemic cardiomyopathy (SCIPIO): initial results of a randomised phase 1 trial," The Lancet, vol. 378, no. 9806, pp. 1847-1857, 2011.

[43] R. R. Makkar, R. R. Smith, K. Cheng et al., "Intracoronary cardiosphere-derived cells for heart regeneration after myocardial infarction (CADUCEUS): a prospective, randomised phase 1 trial," The Lancet, vol. 379, no. 9819, pp. 895904, 2012.

[44] X. L. Tang, G. Rokosh, S. K. Sanganalmath et al., "Intracoronary administration of cardiac progenitor cells alleviates left ventricular dysfunction in rats with a 30-day-old infarction," Circulation, vol. 121, no. 2, pp. 293-305, 2010.

[45] A. M. Smits, L. W. Van Laake, K. Den Ouden et al., "Human cardiomyocyte progenitor cell transplantation preserves longterm function of the infarcted mouse myocardium," Cardiovascular Research, vol. 83, no. 3, pp. 527-535, 2009.

[46] E. Kizana, E. Cingolani, and E. Marbán, "Non-cell-autonomous effects of vector-expressed regulatory RNAs in mammalian heart cells," Gene Therapy, vol. 16, no. 9, pp. 1163$1168,2009$.

[47] P. R. Crisostomo, A. M. Abarbanell, M. Wang, T. Lahm, Y. Wang, and D. R. Meldrum, "Embryonic stem cells attenuate myocardial dysfunction and inflammation after surgical global ischemia via paracrine actions," American Journal of Physiology, vol. 295, no. 4, pp. H1726-H1735, 2008.

[48] M. Gnecchi, H. He, N. Noiseux et al., "Evidence supporting paracrine hypothesis for Akt-modified mesenchymal stem cell-mediated cardiac protection and functional improvement," The FASEB Journal, vol. 20, no. 6, pp. 661-669, 2006.

[49] L. Timmers, S. K. Lim, F. Arslan et al., "Reduction of myocardial infarct size by human mesenchymal stem cell conditioned medium," Stem Cell Research, vol. 1, no. 2, pp. 129-137, 2008.

[50] F. Bönner, N. Borg, S. Burghoff, and J. Schrader, "Resident cardiac immune cells and expression of the ectonucleotidase enzymes CD39 and CD73 after ischemic injury," PLoS One, vol. 7, Article ID e34730, 2012.

[51] S. F. Mause and C. Weber, "Microparticles: protagonists of a novel communication network for intercellular information exchange," Circulation Research, vol. 107, no. 9, pp. 1047-1057, 2010.

[52] C. Théry, "Exosomes: secreted vesicles and intercellular communications," F1000 Biology Reports, vol. 3, no. 1, article 15, 2011.

[53] C. Théry, S. Amigorena, G. Raposo, and A. Clayton, "Isolation and characterization of exosomes from cell culture supernatants and biological fluids," Current Protocols in Cell Biology, vol. 3, article 22, 2006.

[54] S. Mathivanan, H. Ji, and R. J. Simpson, "Exosomes: extracellular organelles important in intercellular communication," Journal of Proteomics, vol. 73, no. 10, pp. 1907-1920, 2010.

[55] P. J. Quesenberry and J. M. Aliotta, "Cellular phenotype switching and microvesicles," Advanced Drug Delivery Reviews, vol. 62, no. 12, pp. 1141-1148, 2010.

[56] S. Mathivanan and R. J. Simpson, "ExoCarta: a compendium of exosomal proteins and RNA," Proteomics, vol. 9, no. 21, pp. 4997-5000, 2009.

[57] M. Ostrowski, N. B. Carmo, S. Krumeich et al., "Rab27a and Rab27b control different steps of the exosome secretion pathway," Nature cell biology, vol. 12, no. 1, pp. 19-3013, 2010.

[58] H. Valadi, K. Ekström, A. Bossios, M. Sjöstrand, J. J. Lee, and J. O. Lötvall, "Exosome-mediated transfer of mRNAs and microRNAs is a novel mechanism of genetic exchange between cells," Nature Cell Biology, vol. 9, no. 6, pp. 654-659, 2007.

[59] J. Ratajczak, K. Miekus, M. Kucia et al., "Embryonic stem cellderived microvesicles reprogram hematopoietic progenitors: evidence for horizontal transfer of mRNA and protein delivery," Leukemia, vol. 20, no. 5, pp. 847-856, 2006. 
[60] K. Ohshima, K. Inoue, A. Fujiwara et al., "Let-7 microRNA family Is selectively secreted into the extracellular environment via exosomes in a metastatic gastric cancer cell line," PLoS ONE, vol. 5, no. 10, Article ID e13247, 2010.

[61] A. Montecalvo, A. T. Larregina, W. J. Shufesky et al., "Mechanism of transfer of functional microRNAs between mouse dendritic cells via exosomes," Blood, vol. 119, no. 3, pp. 756766, 2012.

[62] S. Sahoo, E. Klychko, T. Thorne et al., "Exosomes from human $\mathrm{CD} 4^{+}$stem cells mediate their proangiogenic paracrine activity," Circulation Research, vol. 109, pp. 724-728, 2011.

[63] K. R. Vrijsen, J. P. G. Sluijter, M. W. L. Schuchardt et al., "Cardiomyocyte progenitor cell-derived exosomes stimulate migration of endothelial cells," Journal of Cellular and Molecular Medicine, vol. 14, no. 5, pp. 1064-1070, 2010.

[64] R. C. Lai, T. S. Chen, and S. K. Lim, "Mesenchymal stem cell exosome: a novel stem cell-based therapy for cardiovascular disease," Regenerative Medicine, vol. 6, no. 4, pp. 481-492, 2011.

[65] R. C. Lai, F. Arslan, M. M. Lee et al., "Exosome secreted by MSC reduces myocardial ischemia/reperfusion injury," Stem Cell Research, vol. 4, no. 3, pp. 214-222, 2010.

[66] E. Emmanouilidou, K. Melachroinou, T. Roumeliotis et al., "Cell-produced $\alpha$-synuclein is secreted in a calcium-dependent manner by exosomes and impacts neuronal survival," Journal of Neuroscience, vol. 30, no. 20, pp. 6838-6851, 2010.

[67] P. G. Sreekumar, R. Kannan, M. Kitamura et al., " $\alpha$ B crystallin is apically secreted within exosomes by polarized human retinal pigment epithelium and provides neuroprotection to adjacent cells," PLoS ONE, vol. 5, no. 10, Article ID e12578, 2010.

[68] M. Gherghiceanu and L. M. Popescu, "Cardiac telocytestheir junctions and functional implications," Cell Tissue Research, vol. 348, no. 2, pp. 265-279, 2012.

[69] L. M. Popescu and M. S. Faussone-Pellegrini, “TELOCYTESa case of serendipity: the winding way from Interstitial Cells of Cajal (ICC), via Interstitial Cajal-Like Cells (ICLC) to TELOCYTES," Journal of Cellular and Molecular Medicine, vol. 14, no. 4, pp. 729-740, 2010.

[70] M. Gherghiceanu and L. M. Popescu, "Cardiomyocyte precursors and telocytes in epicardial stem cell niche: electron microscope images," Journal of Cellular and Molecular Medicine, vol. 14, no. 4, pp. 871-877, 2010.

[71] C. G. Manole, V. Cismaşiu, M. Gherghiceanu, and L. M. Popescu, "Experimental acute myocardial infarction: telocytes involvement in neo-angiogenesis," Journal of Cellular and Molecular Medicine, vol. 15, no. 11, pp. 2284-2296, 2011.

[72] H. Yagi, A. Soto-Gutierrez, B. Parekkadan et al., "Mesenchymal stem cells: mechanisms of immunomodulation and homing," Cell Transplantation, vol. 19, no. 6-7, pp. 667-679, 2010.

[73] L. Zitvogel, A. Regnault, A. Lozier et al., "Eradication of established murine tumors using a novel cell-free vaccine: dendritic cell-derived exosomes," Nature Medicine, vol. 4, no. 5, pp. 594-600, 1998.

[74] B. Escudier, T. Dorval, N. Chaput et al., "Vaccination of metastatic melanoma patients with autologous dendritic cell (DC) derived-exosomes: results of the first phase 1 clinical trial," Journal of Translational Medicine, vol. 3, article 10, 2005.

[75] M. A. Morse, J. Garst, T. Osada et al., "A phase I study of dexosome immunotherapy in patients with advanced non-small cell lung cancer," Journal of Translational Medicine, vol. 3, article 9, 2005.

[76] S. Dai, D. Wei, Z. Wu et al., "Phase I clinical trial of autologous ascites-derived exosomes combined with GM-CSF for colorectal cancer," Molecular Therapy, vol. 16, no. 4, pp. 782790,2008 .

[77] K. Malliaras, T. S. Li, D. Luthringer et al., "Safety and efficacy of allogeneic cell therapy in infarcted rats transplanted with mismatched cardiosphere-derived cells," Circulation, vol. 125, pp. 100-112, 2012.

[78] N. Chaput and C. Théry, "Exosomes: immune properties and potential clinical implementations," Seminars in Immunopathology, vol. 33, no. 5, pp. 419-440, 2011. 

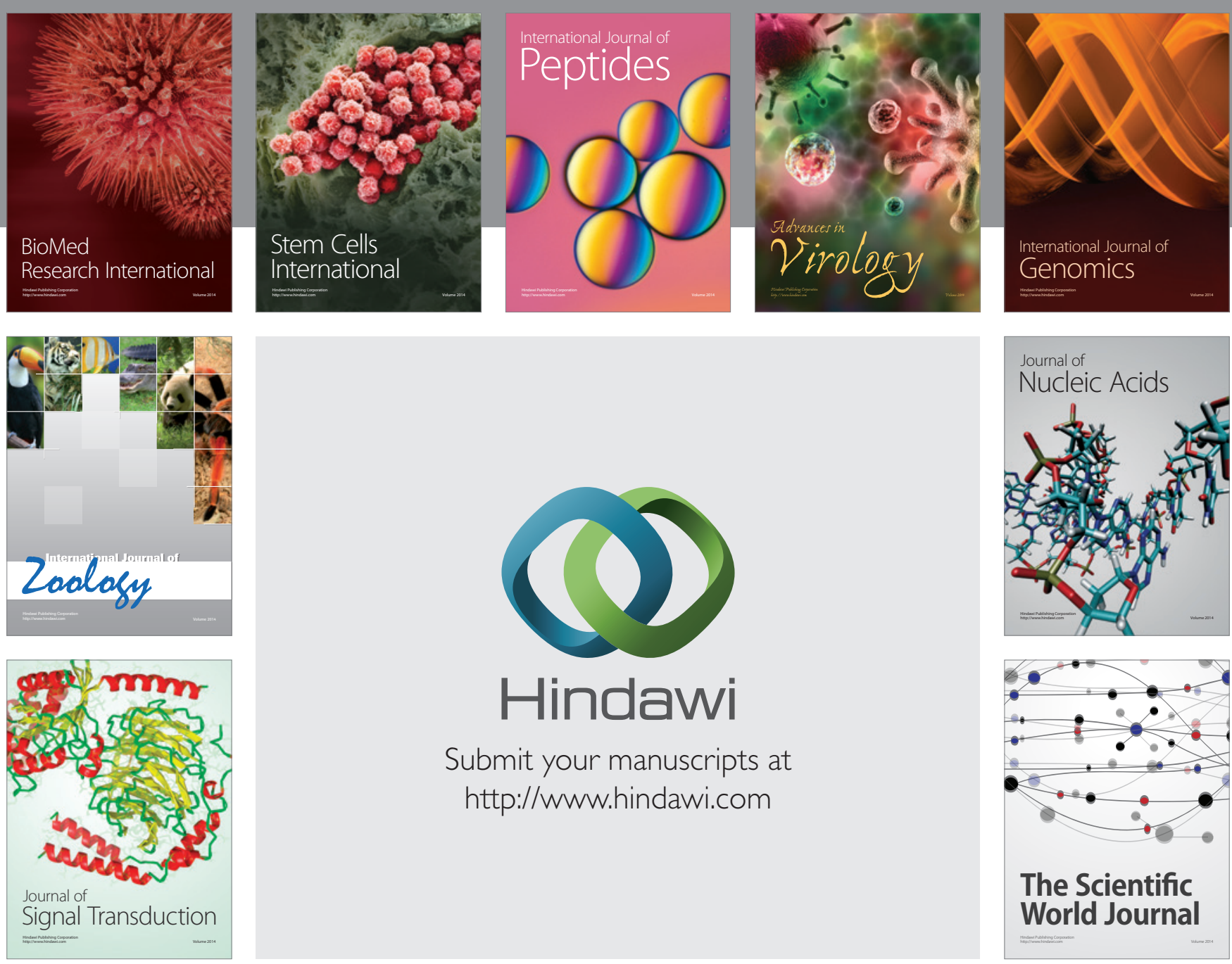

Submit your manuscripts at

http://www.hindawi.com
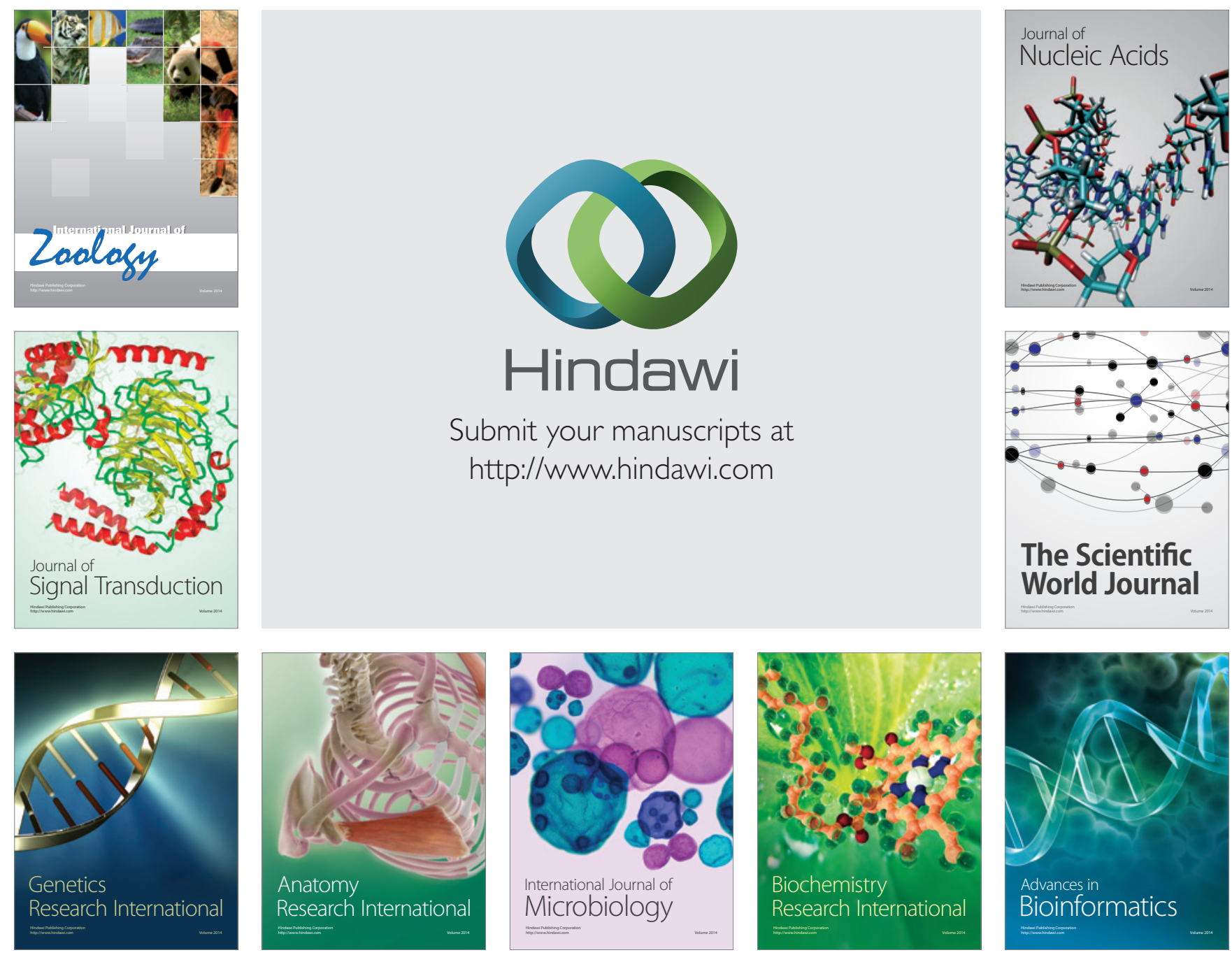

The Scientific World Journal
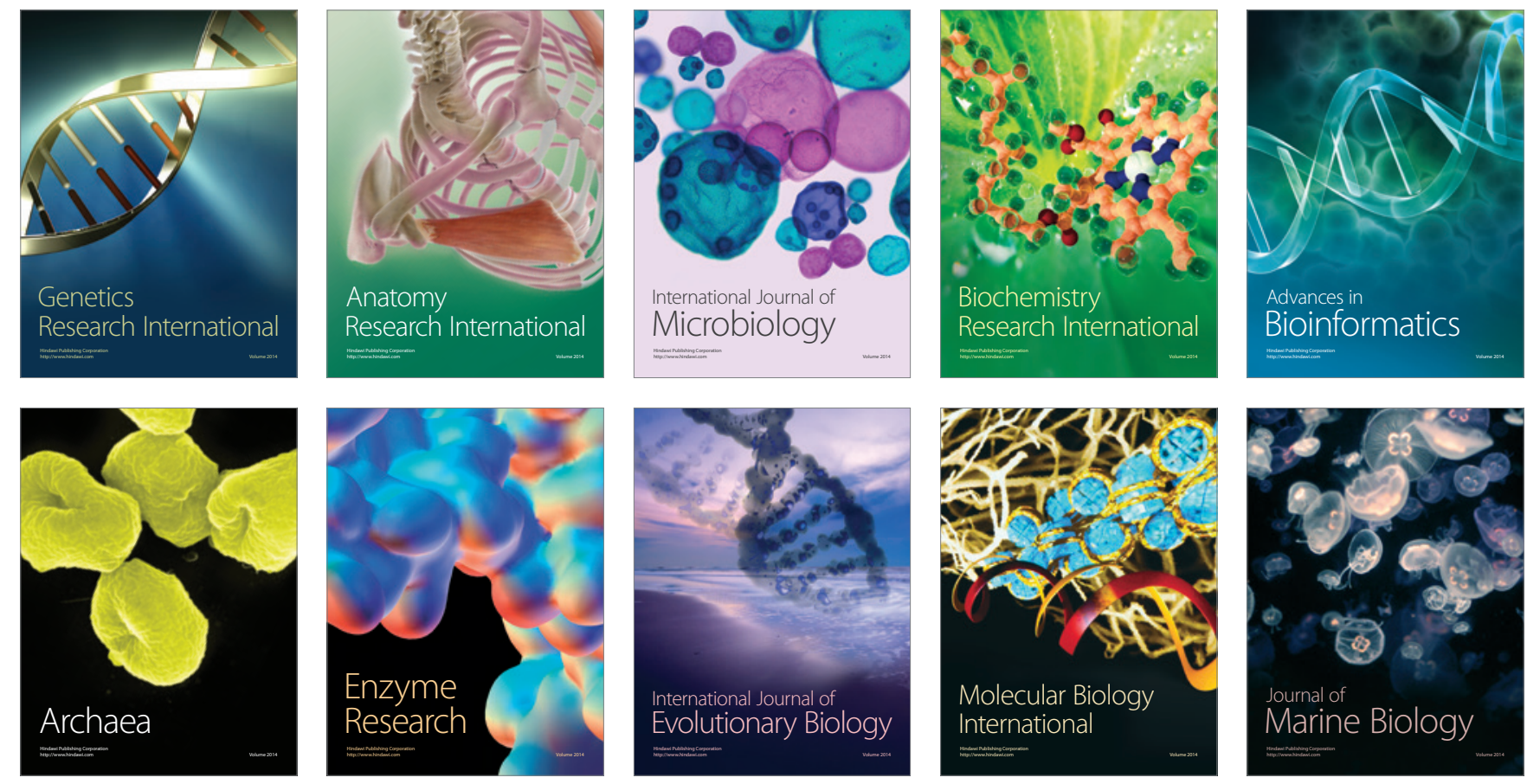